\title{
Negative Pressure versus Conventional Sternal Wound Dressing in Coronary Surgery Using Bilateral Internal Mammary Artery Grafts
}

\author{
Vito Giovanni Ruggieri, MD, PhD, ${ }^{1,2}$ Maud-Emmanuelle Olivier, MD,${ }^{1}$ Chadi Aludaat, MD, ${ }^{1}$ \\ Stefano Rosato, MSc, ${ }^{3}$ Paul Marticho, MD,${ }^{1}$ Yves Assad Saade, MD,${ }^{1}$ Annick Lefebvre, MD ${ }^{4}$ \\ Anne Poncet, MD, ${ }^{5}$ Sylvain Rubin, $\mathrm{MD}, \mathrm{PhD},{ }^{1,2}$ Fausto Biancari, $\mathrm{MD}, \mathrm{PhD}^{6,7}$ \\ ${ }^{1}$ Division of Cardiovascular and Thoracic Surgery, and ${ }^{5}$ Unité d'Anesthésie-Réanimation Cardio-Thoracique, Robert Debré \\ University Hospital, Reims, France; ${ }^{2}$ Université de Reims Champagne Ardenne (URCA), Reims, France; ${ }^{3}$ Center for Global Health, \\ Istituto Superiore di Sanità, Rome, Italy; ${ }^{4}$ Equipe Opérationnelle d'Hygiène, CHU Reims, Hôpital Maison Blanche, Reims, France; \\ ${ }^{6}$ Heart Center, Turku University Hospital and Department of Surgery, University of Turku, Turku, Finland, ${ }^{7}$ Department of Surgery, \\ University of Oulu, Oulu, Finland
}

\section{ABSTRACT}

Background: Sternal wound infection (SWI) is a major complication occurring often after coronary artery bypass grafting (CABG) using bilateral internal mammary artery (BIMA) grafts. The aim of this study is to assess whether such a risk may be reduced by using incision negative pressure wound therapy (INPWT).

Methods: Data on patients undergoing isolated CABG using BIMA grafts at the Reims University Hospital, France, from 2013 to 2016 without or with INPWT was prospectively collected.

Results: INPWT was used in 161 patients and conventional sterile wound dressing was used in 266 patients. Propensity score matching resulted in 128 pairs with similar characteristics. SWIs were similarly distributed between the conventional sterile wound dressing $(10.9 \%)$ and the INPWT cohorts $(10.2 \%)(P=1.00)$. Patients treated with INPWT had a lower rate of deep SWI/mediastinitis than patients who had conventional sterile dressing $(5.5 \%$ versus $10.2 \%, P=.210$ ), but the difference did not reach statistical significance. Tests for interaction confirmed these findings in different patient subgroups.

Conclusion: The routine use of INPWT may not significantly reduce the risk of SWI in patients undergoing BIMA grafting. In view of previous reports showing a benefit with the use of this method, a large randomized study is justified to assess the efficacy of INPWT in patients undergoing cardiac surgery.

\section{INTRODUCTION}

Sternal wound infection (SWI) is one of the most severe complications occurring after coronary artery bypass grafting

Received October 28, 2018; accepted December 12, 2018.

Correspondence: Prof. Vito Giovanni Ruggieri, Division of Cardiovascular and Thoracic Surgery, Robert Debré University Hospital, Reims, France; +33637902593 (e-mail:vgruggieri@chu-reims.fr).
(CABG). In particular, SWI is the Achilles' heel of bilateral internal mammary artery (BIMA) grafting [Kouchoukos 1990] as harvesting of BIMA grafts causes a significant reduction of blood supply to the sternum [Parish 1992]. Although BIMA grafting may improve the late outcome after CABG [Lytle 1999], the fear of SWI is one of the reasons preventing the widespread use of this revascularization strategy [Mastrobuoni 2012]. A number of pre-, intra- and postoperative strategies are known to potentially reduce the risk of SWI [Sajja 2015]. Recently, incisional negative pressure wound therapy (INPWT) on the closed sternal wound has been introduced as a prophylactic measure to reduce the risk of SWI with encouraging results [Grauhan 2014]. The mechanism behind the potential efficacy of INPWT is the removal of fluids and infectious material from the surgical incision. The efficacy of this method to prevent sternal wound complications in patients undergoing BIMA grafting has been evaluated in this study.

\section{MATERIALS AND METHODS}

\section{Patient Population and Inclusion/Exclusion Criteria}

427 consecutive patients underwent isolated CABG using BIMA grafts at the Reims University Hospital, Reims, France, from April 2013 to December 2016. This study was approved by the Institutional Review Board of this institution. Data on baseline characteristics, operative variables, and outcomes of these patients were prospectively collected into a dedicated datasheet. Patients who underwent CABG using a single internal mammary artery graft or any associated major cardiac procedure were excluded from this study.

\section{Skin Disinfection, Antibiotic Prophylaxis, and Surgical Technique}

Preoperative screening of oropharyngeal bacterial contamination was not performed in these patients. Cefazolin $1.5 \mathrm{gr}$ was administered for antibiotic prophylaxis before anesthesia induction followed by cefazolin $750 \mathrm{mg}$ every 2 hours intraoperatively. Furthermore, cefazolin $750 \mathrm{mg}$ was 
Table 1. Baseline Characteristics in the Overall Series and Propensity Score Matched Pairs



Continuous variables are reported as the mean and standard deviation. Nominal variables are reported as counts and percentages (in parentheses). INPWT indicates incisional negative pressure wound therapy; BMI, body mass index; LVEF, left ventricular ejection fraction; XCT, cross-clamping time; CPBT, cardiopulmonary bypass time.

administered every 6 hours for 48 hours after surgery. The surgical field was treated in the operating room immediately before surgery with povidone-iodine $7.5 \%$ (Betadine Surgical Scrub, Meda Pharma, Paris, France) four times followed by washing with povidone-iodine $10 \%$ once (Betadine Dermique, Meda Pharma, Paris, France). No topical antibiotics on the sternal wound were used; no antibiotic impregnated collagen agents were used in this series. Bone wax was used in all patients.

BIMA grafts were harvested with an intact surrounding pedicle using diathermy. Sternotomy was closed with seven single metallic-wire cerclages. The subcutaneous layer was closed with Vicryl 1-0 or 2-0 (Ethicon SAS, Issy Les Moulineaux, France) and the skin was closed intracutaneously with Monocryl 4-0 (Ethicon SAS, Issy Les Moulineaux, France). Blood glucose was tightly monitored and intravenous insulin routinely administered in diabetics after surgery.

\section{Sternal Wound Therapy}

During the study period, a closed incision negative pressure dressing (Prevena, Kinetic Concepts, San Antonio, TX, USA) was used on the closed sternal wound in 161 patients. The remaining patients were managed with a conventional sterile wound dressing. The decision to use INPWT was based on the individual surgeon's choice. The prevalence of the use of this negative pressure dressing was $9.1 \%$ in 2013 , $24.1 \%$ in $2014,51.7 \%$ in 2015 , and $42.9 \%$ in $2016(P<.001)$.

In the INPWT cohort, the sternal wound was covered with this dressing immediately after skin closure under sterile conditions in the operating room. The suction pump was connected
Table 2. Results of Cultures in 38 Patients with Postoperative Sternal Wound Infection

\begin{tabular}{ll}
\hline Bacterial strains* & $\mathrm{n}$ \\
\hline Staphylococcus aureus & 11 \\
Staphylococcus epidermidis & 9 \\
Staphycoccus coag. neg. & 9 \\
Escherichia coli & 4 \\
Serratia marcescens & 2 \\
Propionibacterium acnes & 2 \\
Staphylococcus schleiferi & 1 \\
Proteus mirabilis & 1 \\
Morganella morgani & 1 \\
Klebsiella pneumoniae & 1 \\
Enterobacter cloacae & 1 \\
Enterobacter aerogenes & 1 \\
Achromobacter & 1 \\
Gram positive bacteria & 1 \\
No bacterial growth & 1 \\
\hline
\end{tabular}

*More than one bacterial strain was observed in seven patients.

and a negative pressure of $-125 \mathrm{mmHg}$ was applied. This foam dressing was removed after 5 or 7 days under medical 
Table 3. Outcomes

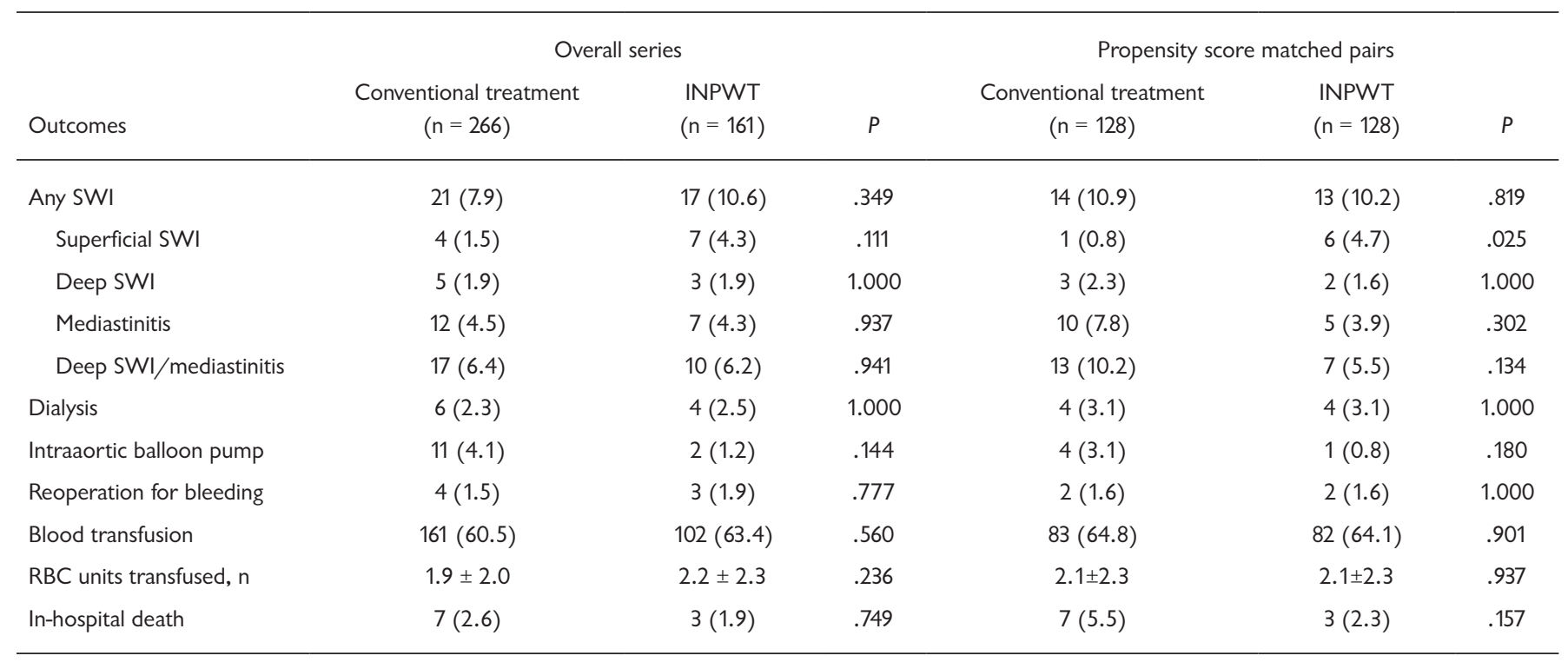

Continuous variables are reported as the mean and standard deviation. Nominal variables are reported as counts and percentages (in parentheses). INPWT indicates incisional negative pressure wound therapy; SWI, sternal wound infection; RBC, red blood cell; ICU, intensive care unit.

supervision. In the control cohort, a conventional wound dressing was used (Tegaderm, 3M, St. Paul, MN, USA). The first wound dressing was changed on the second postoperative day and the sternal wound was inspected every 2 days.

\section{Outcome Measures}

The primary outcome of this study was SWI of any severity as well as superficial SWI, deep SWI, and mediastinitis. The diagnosis and severity of SWI were made and graded according to the Centers for Disease Control and Prevention criteria of surgical site infections [Mangram 1999]. Secondary outcomes of this study were in-hospital death, dialysis, postoperative intraaortic balloon pump, blood transfusion, and reoperation for bleeding.

\section{Statistical Analysis}

Statistical analysis was performed using the SAS statistical package, version 9.2 (SAS Institute, Cary, NC, USA) and the SPSS version 24.0 statistical software (IBM Corporation, New York, New York, USA). No attempt to replace missing values was made. Mann-Whitney $U$ test, Fisher exact test, and Pearson chi-square test were used for univariate analysis in the unmatched population. A propensity score matching was employed to select two groups of patients undergoing INPWT or conventional sterile wound dressing, respectively, with similar baseline characteristics. The propensity score was estimated using a non-parsimonious logistic regression model including the following covariates: age, sex, body mass index, pulmonary disease, diabetes, prior cardiac surgery, left ventricular ejection fraction, EuroSCORE II [Nashef 2012], and STS score [Online STS Adult Cardiac Surgery].

One-to-one propensity score matching was performed employing the nearest neighbor method and a caliper of 0.2 of the standard deviation of the logit of the propensity score. To evaluate the balance between the matched groups, the t-test for paired samples for continuous variables, the McNemar test for dichotomous variables and the analysis of the standardized differences after matching were used. The imbalance between the study cohorts was acceptable when standardized difference was less than $10 \%$. Tests for interaction for any SWI as well as deep SWI/mediastinitis were performed by subgroups with relevant comorbidities. All tests were two-sided and $P<$ .05 was considered statistically significant.

\section{RESULTS}

Patients' characteristics and operative variables are summarized in Table 1 . In this series, 38 patients experienced SWI (8.9\%). Superficial SWI was observed in 11 patients $(2.6 \%)$ and deep SWI/mediastinitis in 27 patients $(6.3 \%$; mediastinitis in 19 patients [4.4\%]). Culture growth was positive in all cases (Table 2), but one patient had otherwise clear clinical signs of superficial SWI.

In the overall series, no significant differences were observed between these cohorts either in terms of primary or secondary outcomes (Table 3 ).

Propensity score matching resulted in 128 pairs with similar baseline characteristics. Both EuroSCORE 2 and STS score were similar between the study cohorts as indicated by standardized differences $<10 \%$.

McNemar test showed that SWIs (conventional sterile wound dressing $10.9 \%$ versus INPWT $10.2 \%, P=1.00$ ) were similarly distributed between the conventional and the INPWT cohorts (Table 3). Patients treated with INPWT had numerically less deep SWI/mediastinitis than control patients 
Table 4. Sternal Wound Infection and Tests for Interaction by Subgroups

\begin{tabular}{lcccccc}
\hline & & & & \multicolumn{2}{c}{ Interaction } \\
& OR & $95 \% \mathrm{Cl}$ & & $z$ & $P$ \\
\hline $\begin{array}{l}\text { INPWT versus con- } \\
\text { ventional treatment }\end{array}$ & 0.92 & 0.45 & 1.87 & & \\
Male & 0.73 & 0.32 & 1.63 & 1.24 & .215 \\
Female & 2.25 & 0.46 & 11.05 & & \\
Age $<70$ y & 0.86 & 0.33 & 2.26 & 0.20 & .838 \\
Age $\geq 70$ y & 1.00 & 0.35 & 2.84 & & \\
No diabetes & 0.65 & 0.10 & 4.25 & 0.41 & .682 \\
Diabetes & 1.00 & 0.46 & 2.19 & & \\
No pulmonary disease & 0.90 & 0.40 & 2.01 & 0.12 & .903 \\
Pulmonary disease & 1.00 & 0.22 & 4.54 & & \\
LVEF $>50 \%$ & 1.90 & 0.54 & 6.73 & -1.54 & .125 \\
LVEF $\leq 50 \%$ & 0.57 & 0.24 & 1.37 & & \\
BMI <30 & 0.79 & 0.23 & 2.70 & 0.30 & .761 \\
BMI $\geq 30$ & 1.00 & 0.40 & 2.48 & & \\
\hline
\end{tabular}

INPWT indicates incisional negative pressure wound therapy; OR, odds ratio; $\mathrm{Cl}$, confidence interval; LVEF, left ventricular ejection fraction; $\mathrm{BMI}$, body mass index.

(5.5\% versus $10.2 \%, P=0.210$ ), but the difference did not reach statistical significance. This difference was related to a non-statistically significant lower rate of mediastinitis in the INPWT cohort (3.9\% versus $7.8 \%, P=.267)$.

Tests for interaction showed that these findings were consistent for any SWI as well as deep SWI/mediastinitis among patients with and without significant comorbidities (Tables 4 and 5).

No differences were observed in other outcomes between the study cohorts (Table 3).

\section{DISCUSSION}

The present results showed that using INPWT does not significantly reduce the risk of SWI in patients undergoing CABG with the use of BIMA grafts. These findings are of clinical relevance since these patients are at formidable risk of sternal wound complications, which in turn prevents the widespread use of BIMA grafting. Despite the encouraging early reports by Atkins et al [Atkins 2009] and Colli and Camara [Colli 2011], we were not able to observe any significant benefit with the use of this new prophylactic wound treatment. Interaction analysis showed that the benefit of INPWT was not evident even in subgroups of patients with comorbidities of clinical relevance. Previous comparative studies by Grauhan and colleagues [Grauhan 2013; Grauhan 2014] showed a decreased risk of SWI after adult cardiac surgery. One of these studies was lacking any baseline data on these patients
Table 5. Deep Sternal Wound Infection or Mediastinitis and Tests for Interaction by Subgroups

\begin{tabular}{|c|c|c|c|c|c|}
\hline & \multirow[b]{2}{*}{ OR } & \multirow[b]{2}{*}{$95 \% \mathrm{Cl}$} & & \multicolumn{2}{|c|}{ Interaction } \\
\hline & & & & Chi square & $P$ \\
\hline $\begin{array}{l}\text { INPWT versus con- } \\
\text { ventional treatment }\end{array}$ & 0.51 & 0.21 & 1.24 & & \\
\hline Male & 0.43 & 0.15 & 1.23 & 0.89 & .3731 \\
\hline Female & 1.00 & 0.21 & 4.66 & & \\
\hline Age $<70$ years & 0.41 & 0.10 & 1.70 & 0.50 & .6184 \\
\hline Age $\geq 70$ years & 0.64 & 0.22 & 1.88 & & \\
\hline No diabetes & 0.32 & 0.03 & 3.29 & 0.44 & .6614 \\
\hline Diabetes & 0.56 & 0.21 & 1.50 & & \\
\hline No pulmonary disease & 0.38 & 0.12 & 1.17 & 1.01 & .3112 \\
\hline Pulmonary disease & 1.00 & 0.22 & 4.54 & & \\
\hline LVEF > $>50 \%$ & 1.28 & 0.35 & 4.69 & -1.87 & .0620 \\
\hline LVEF $\leq 50 \%$ & 0.20 & 0.05 & 0.85 & & \\
\hline Body mass index $<30$ & 0.74 & 0.15 & 3.55 & -0.63 & .5266 \\
\hline Body mass index $\geq 30$ & 0.40 & 0.13 & 1.19 & & \\
\hline
\end{tabular}

INPWT indicates incisional negative pressure wound therapy; OR, odds ratio; $\mathrm{Cl}$, confidence interval; LVEF, left ventricular ejection fraction.

and comparative analysis was not adjusted for baseline and operative covariates [Grauhan 2014]. Another study including obese patients showed that the risk of SWI was significantly lower after INPWT compared to conventional sterile wound dressing (4\% versus $16 \%, P=.03$ ) [Grauhan 2013]. The present study showed that INPWT was associated with a numerically lower number of severe SWI, i.e. deep SWI and mediastinitis, compared to the conventional sterile wound treatment. Although this difference did not reach statistical significance, this observation suggests that further studies with adequate sample size are needed to get conclusive results on the potential efficacy of INPWT in patients undergoing adult cardiac surgery. In fact, in order to detect a $50 \%$ reduction of the risk of deep SWI/mediastinitis, the estimated sample size for matched case-control study would be 255 patients in each cohort (estimation based on the herein observed event rate in the control group of $10 \%$, alpha $=0.05$, power $=0.80$ ). Therefore, post-hoc analysis suggests that the present analysis is underpowered to detect such a significant risk reduction. However, randomization to INPWT and conventional sterile wound treatment of 510 patients undergoing BIMA grafting is unrealistic. An even larger study population would be necessary to demonstrate a significant decrease of the risk of deep SWI with INPWT when a pooled rate of $2.4 \%$ of this complication [Deo 2015] is considered. Importantly, a costanalysis is needed as well to estimate the economic burden for an expected large number of patients in need of treatment to prevent one surgical site infection.

The limited number and suboptimal quality of studies evaluating INPWT in adult cardiac surgery prevents conclusive 
results in this setting. However, the potential benefits of INPWT could be extrapolated from the results of randomized studies in other fields of surgery. Two recent randomized studies showed a significant benefit of using INPWT after laparotomy [O'Leary 2017; Li 2017]. However, a much larger randomized trial by Shen et al [Shen 2017] showed no benefit with the use of INPWT in patients undergoing abdominal surgery. Similarly, INPWT failed to reduce the risk of surgical site infection in other randomized studies in different surgical settings [Manoharan 2016; Masden 2012]. These findings suggest that the value of INPWT after surgery is still controversial and its routine use cannot be recommended until adequately powered trials have demonstrated its efficacy in reducing surgical wound complications in patients undergoing adult cardiac surgery. Pooled analyses of matched casecontrol series may also be valuable to better assess the efficacy of INPWT in these patients.

\section{Study Limitations}

The non-randomized nature is a major limitation of this study. However, data retrieval was prospectively performed and data on SWI, bacterial culture findings, as well as medical and surgical treatment of these patients was detailed according to pre-fixed criteria. Furthermore, this study is not adequately powered to detect a $50 \%$ reduction of the risk based on the proportion of SWIs observed in the conventional sterile wound dressing cohort.

\section{Conclusion}

The results of this study suggest that routine use of negative pressure management may not significantly reduce the risk of SWI in patients undergoing CABG with the use of BIMA grafts. In view of previous reports which showed a significant benefit with the use of this method and the herein observed non-statistically significant lower rate of deep SWI/ mediastinitis, a large randomized study is justified to definitely assess the efficacy of INPWT in patients undergoing adult cardiac surgery. Pooled analyses of matched case-control series of good quality may also be valuable to further assess the efficacy of INPWT in these patients.

\section{REFERENCES}

Atkins BZ, Wooten MK, Kistler J, et al. 2009. Does negative pressure wound therapy have a role in preventing poststernotomy wound complications? Surg Innov 16:140-6.

Colli A, Camara ML. 2011 First experience with a new negative pressure incision management system on surgical incisions after cardiac surgery in high risk patients. J Cardiothorac Surg 6:160.
Deo SV, Altarabsheh SE, Shah IK, et al. 2015. Are two really always better than one? Results, concerns and controversies in the use of bilateral internal thoracic arteries for coronary artery bypass grafting in the elderly: a systematic review and meta-analysis. Int J Surg 16:Pt B,163-70.

Grauhan O, Navasardyan A, Hofmann M, et al. 2013. Prevention of poststernotomy wound infections in obese patients by negative pressure wound therapy. J Thorac Cardiovasc Surg 145:1387-92.

Grauhan O, Navasardyan A, Tutkun B, et al. 2014. Effect of surgical incision management on wound infections in a poststernotomy patient population. Int Wound J 11:suppl 1,6-9. Kouchoukos NT, Wareing TH, Murphy SF, et al. 1990. Risks of bilateral internal mammary artery bypass grafting. Ann Thorac Surg 49:210-17.

Li PY, Yang D, Liu D, et al. 2017. Reducing surgical site infection with negative-pressure wound therapy after open abdominal surgery: a prospective randomized controlled study. Scand J Surg 106:189-95.

Lytle BW, Blackstone EH, Loop FD, et al. 1999. Two internal thoracic artery grafts are better than one. J Thorac Cardiovasc Surg 117:855-72.

Mangram AJ, Horan TC, Pearson ML, et al. 1999. Guideline for prevention of surgical site infection, 1999. Hospital Infection Control Practices Advisory Committee. Infect Control Hosp Epidemiol 20:250-78.

Manoharan V, Grant AL, Harris AC, et al. 2016. Closed incision negative pressure wound therapy vs conventional dry dressings after primary knee arthroplasty: a randomized controlled study. J Arthroplasty 31:2487-94.

Masden D, Goldstein J, Endara M, et al. 2012. Negative pressure wound therapy for at-risk surgical closures in patients with multiple comorbidities: a prospective randomized controlled study. Ann Surg 255:1043-7.

Mastrobuoni S, Gawad N, Price J, et al. 2012. Use of bilateral internal thoracic artery during coronary artery bypass graft surgery in Canada: the bilateral internal thoracic artery survey. J Thorac Cardiovasc Surg 144:874-9.

Nashef SA, Roques F, Sharples LD, et al. 2012. EuroSCORE II. Eur J Cardiothorac Surg 41:734-44.

O'Leary DP, Peirce C, Anglim B, et al. 2017. Prophylactic negative pressure dressing use in closed laparotomy wounds following abdominal operations: a randomized, controlled, open-label trial: The P.I.C.O. Trial. Ann Surg 265:1082-6.

Online STS Adult Cardiac Surgery Risk Calculator. http://riskcalc.sts. org/stswebriskcalc/\#/. [Accessed June 2017].

Parish MA, Asai T, Grossi EA, et al. 1992. The effects of different techniques of internal mammary artery harvesting on sternal blood flow. J Thorac Cardiovasc Surg 104:1303-7.

Sajja LR. 2015. Strategies to reduce deep sternal wound infection after bilateral internal mammary artery grafting. Int J Surg 16:Pt B,171-8.

Shen P, Blackham AU, Lewis S, et al. 2017. Phase II randomized trial of negative-pressure wound therapy to decrease surgical site infection in patients undergoing laparotomy for gastrointestinal, pancreatic, and peritoneal surface malignancies. J Am Coll Surg 224:726-37. 